\title{
AN ACCURATE FINGERPRINT REFERENCE POINT DETERMINATION METHOD BASED ON CURVATURE ESTIMATION OF SEPARATED RIDGES
}

\author{
RAFAL DOROZ $^{a, *}$, KRZYSZTOF WROBEL $^{a}$, PIOTR PORWIK $^{a}$ \\ ${ }^{a}$ Institute of Computer Science \\ University of Silesia, ul. Będzinska 39, 41-200 Sosnowiec, Poland \\ e-mail: rafal.doroz@us.edu.pl
}

\begin{abstract}
This paper presents an effective method for the detection of a fingerprint's reference point by analyzing fingerprint ridges' curvatures. The proposed approach is a multi-stage system. The first step extracts the fingerprint ridges from an image and transforms them into chains of discrete points. In the second step, the obtained chains of points are processed by a dedicated algorithm to detect corners and other points of highest curvature on their planar surface. In a series of experiments we demonstrate that the proposed method based on this algorithm allows effective determination of fingerprint reference points. Furthermore, the proposed method is relatively simple and achieves better results when compared with the approaches known from the literature. The reference point detection experiments were conducted using publicly available fingerprint databases FVC2000, FVC2002, FVC2004 and NIST.
\end{abstract}

Keywords: biometrics, image processing, fingerprint recognition, Kolmogorov-Smirnov statistical test, reference point.

\section{Introduction}

Recognition systems, classifiers, as well as self-adaptive classifiers (Porwik et al., 2016; Krawczyk and Woźniak, 2016), are developed and applied in many domains, e.g., electronics, biometrics (Putz-Leszczyńska, 2015; Pujol et al., 2016), medicine (Porwik et al., 2009; Porwik and Doroz, 2014; Koprowski, 2016; Kowal and Filipczuk, 2014; Mazurek and Oszutowska-Mazurek, 2014). Of the many biometric techniques, fingerprint identification is most prevalent, be it as a tool in police work and the courts, or in a range of commercial applications: banking, security systems, etc. Fingerprints, or fingermarks, refer to the impressions left on various surfaces due to contact with a given person's fingertips. The fingerprint image is a system of oriented texture and includes important personal structural information. The fingerprint consists of line patterns called 'ridges' and 'furrows' (valleys). On the fingerprint surface we can generally point out ridges, 'minutiae points' (a ridge bifurcation or a ridge ending), as well as fine features such as sweat pores, amongst other intra-ridge details (Jain et al., 2007).

A captured fingerprint also contains two important

${ }^{*}$ Corresponding author areas, where 'core' and 'delta' points (also referred to as 'singular' points) are located. In most cases, a core point is identified as a reference point (Srinivasan and Murthy, 1992; Galar et al., 2015). It should be noted that these points are always strongly stable and also scale invariant, so singular points are the most important global characteristics of a fingerprint. Singular points are located in the regions where the ridge curvature is highest and where the direction of the ridges is changing rapidly (Bo et al., 2008). The delta area is a triangular area where the ridges radiate outward in three directions (Bo et al., 2008). Point pattern matching methods become easier if the core and delta points are known (Srinivasan and Murthy, 1992; Zacharias et al., 2017).

Fingerprints are identified by examining and comparing the minutiae characteristics of two different finger impressions to determine if these characteristics occupy the same relative area and position. In biometrics, minutiae points of different types represents unique, major features of a fingerprint, e.g., ridge endings, bifurcations, islands, etc. These features are each an element of fingerprint-based biometric recognition systems. Thus, some landmarks (here singular points) on each fingerprint image should be initially determined. 
The reference point is employed in those fingerprint comparison techniques where the given template and an unknown fingerprint image have to be matched. It should be noted that a large number of classification and matching algorithms can be used for such operations (Galar et al., 2015; Bo et al., 2008; Jain et al., 2000; Porwik and Wieclaw, 2004; Khalil, 2015; Xie and Zhang, 2016; Weng et al., 2011; Gavrilova and Monwar, 2013).

In practice, two types of core points can occur: concave and convex (Le and Van, 2012; Arjona et al., 2011). A core point located on a convex curving ridge is called a convex core point, whereas a core point located on a concave curving ridge will be called a concave core point. Based on these core points, the fingerprint's reference point can be identified. Also, on the finger's surface, a delta point can be observed. This point lies on a friction ridge at or nearest to the point of divergence of two lines. The core and delta points are depicted in Fig.1.

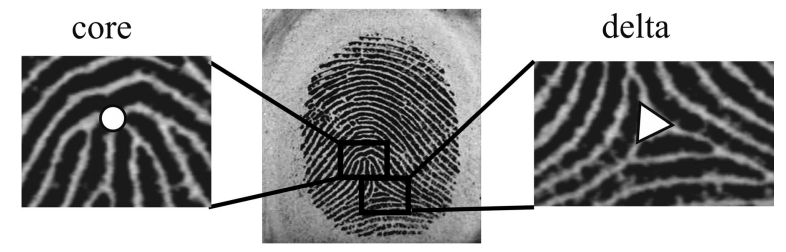

Fig. 1. Characteristic fingerprint areas with a concave-type core and a delta point.

It should be noted that, on a finger's surface, various combinations of core and delta points may be displayed. For example, the following combinations of singular points can appear on the finger's skin: one convex and one delta point, or convex-concave points with two deltas, or one convex and one concave point along with one delta point (Arjona et al., 2011). This observation follows from the distribution of fingerprint classes over the population, where the most popular classes are 'arch', 'tend arch', 'right loop', 'left loop' and 'whorl' (Galar et al., 2015; Porwik and Wieclaw, 2004). In practice, the reference point is defined as the upper-most point of the inner-most ridge of the loop, that is, the point to which ridges tend to converge (Galar et al., 2015). This observation follows from the population fingerprint class distribution. In many instances, this assumption simplifies reference point detection.

In some cases, core and delta point pairs may be detected incorrectly in a pattern area. Moreover, fingerprint recognition algorithms should be unaffected by the rotation and translation of fingerprint images. These troubles and objects diversity follow from the finger skin's morphology, because the number of core and delta points varies in different types of fingerprints (Galar et al., 2015; Arjona et al., 2011). Due to these diversities, the credible detection of reference points for various types of fingerprints still remains a challenge and, despite the importance of singular point extraction, is still an open problem.

The main contributions of our method are as follows:

- a novel strategy for identifying fingerprint ridges' separations,

- a proposition of a new fingerprint ridges curvature determination method,

- introduction of the effective Kolmogorov-Smirnov outlier elimination method,

- a comparison of our methodology with other state-of-the-art approaches,

- a comparison of our results achieved over various datasets and fingerprint acquisition devices.

\section{Related work}

Simple and multi-criteria reference point localization algorithms have a long tradition in the numerous core-point detection methods reported in the literature. Many reference point detection algorithms utilize structural information taken from a directional image. A majority of the approaches proposed so far for singularity detection operate on the basis of ridge orientation images and orientation-consistency-based techniques (Maltoni, 2009).

Orientation maps (OMs) represent the local ridge flow in a fingerprint image (Galar et al., 2015; Jirachaweng et al., 2011; Wang et al., 2007; Gupta and Gupta, 2016). The importance of OMs comes from the fact that these objects are used in almost all biometric-based fingerprints analyses. Unfortunately, an $\mathrm{OM}$ may generate too many singular points such that some are detected incorrectly. Other methods use techniques based on Poincaré index determinations, or friction ridges masks, and many other aforementioned techniques that incorporate dedicated approaches based on heuristic image processing techniques (Porwik and Wieclaw, 2004; Khalil, 2015; Le and Van, 2012; Arjona et al., 2011; Wang et al., 2007).

Another issue related to the singularity detection problem is the quality of the fingerprints. Images with low contrast or only partially defined cannot be processed correctly. For his reason, various preprocessing methods are needed in order to raise the quality of fingerprint images. Nowadays, in serious investigations, the most promising approaches are heterogeneous techniques that combine advance mathematical models with image processing techniques. These allow the use of fingertips' natural features (ridges, valleys, minutiae) as well as the use of new features extracted from a processed primary fingerprint. 
Others (Srinivasan and Murthy, 1992) proposed a local orientation histogram based on block directional images for the detection of singular points. A fingerprint's directional histograms are resistant to the presence of noise in the input image. Unfortunately, this segmentation-based method suffers from a loss of ridge details.

The Poincaré approach is a well-known technique for singular point detection (and not only in the domain of fingerprint recognition) (Bo et al., 2008; Bazen and Gerez, 2002). A method based on the Poincaré index can be used for fingerprint singularity detection whenever a fingerprint has a well-defined orientation. Unfortunately, this approach can generate misjudged singulars when supplied with low quality images and in those cases when ridges direction is near to $\pm \pi / 2$ (Le and Van, 2012). Additionally, and significantly, this algorithm has a high computational complexity. A traditional Poincaré-based method can also imprecisely locate singular points whenever a fingerprint map features skin cracks, scars or dermatosis. Consequently, such a method requires additional treatment to improve the quality of singularity determination. Due to these limitations of the Poincaré method, a different approach to reference point detection and fingerprints matching has been proposed (Jain et al., 2000). The authors of that work introduced an analysis of the neighborhood pixels around any place which might be a reference point. This was done by the computation of the sine components of the orientation field. Finally, a gray-scale map of the fingerprint is generated such that the single darkest pixel marks the coordinates of the detected reference point. Unfortunately, this approach does not overcome the limitations mentioned above: singularities cannot be detected when they are located near to the image's border.

Le and Van (2012), Liu et al. (2005) and Jain et al. (2000) improved the orientation smoothing method based on an adaptive neighborhood in order to localize the reference point on the fingerprint. The effectiveness of these filters was improved based on changes in ridge orientation consistency, allowing a more precise determination of those places with the highest ridge curvature. This method is very efficient. However, searching for the highest ridge curvature is only based on an estimation of the orientation field, so that, in some cases, the curvature computation can be unreliable. In addition, the proposed approach has a high computation cost and is sensitive to interference. Le and Van (2012) as well as Liu et al. (2005) proposed techniques that were checked by only one single fingerprint database, so the results were somewhat limited.

From the work of Jin and Kim (2010) it follows that singular points can be extracted from a multi-scale Gaussian filtered orientation field in such a way as to achieve pixel-level accuracy. Thus, this method is similar to the idea presented by Jain et al. (2000).

Porwik and Wieclaw (2004) presented an approach based on an $\mathrm{OM}$ and supported by a fixed number of 'identification masks.' These masks reflect possible slopes of the friction ridges on the OM image. In the worst case, each identification mask can reveal different reference points so that, finally, on the basis of influence rules, one reference point can be selected. The main drawback of this method is the problem with the identification of reference points in those cases when these points are located near the image border. Additionally, this method becomes less effective when the resolution of the input images changes.

The author of the reference point localization work, Khalil (2015), proposed an algorithm based on a discrete wavelet transform. While this is very productive method, the author's camera images had some illumination problems due to the use of several lighting sources.

\section{Proposed method: The general idea}

In this paper, in contrast to the approaches briefly presented in the previous section, a new and simpler strategy of reference point detection will be introduced. In the proposed method, the reference point will be defined as the point on the fingerprint ridge with the maximum curvature, which is usually located in the central area of the fingerprint. Our strategy is composed of several steps.

- If the fingerprint image quality is low, then the image quality must first be improved. The enhancement procedure involves increasing the contrast between the ridges and valleys, and ridges' false broken points generated during the image enrollment process are reconnected. The image quality is achieved via the method presented by Porwik and Wieclaw (2008).

- In the next step, the image is converted into a binary representation and friction ridges are thinned by the algorithm of Pavlidis (1982) or Tabedzki et al. (2016). In our paper we employ Pavlidis's method. Consequently, all fingerprint lines in the binary image have a thickness of only one pixel.

- By means of the proposed Algorithm 1 the friction ridges are atomized. This means that each ridge is separately extracted from the digital fingerprint image. This algorithm automatically transforms all ridges into ordered chains of discrete points. Additionally, in each chain the first and the last points of the chain are appropriately labeled.

- In the subsequent step, the highest curvature of each single ridge is determined by an analysis of the chain, of points lying on this friction ridge. This analysis is conducted as a separate dedicated procedure. 
- Due to the anatomical properties of human fingerprints, different ridge points can have similar curvatures, but only one of these should be identified as a reference point. All inappropriate points have to be efficiently recognized and removed. For example, deltas, bifurcation points (see Fig. 1) as well as points located in inappropriate ridge fingerprint structures (see Figs. 9 and 10) need to be eliminated. Points identified as outliers and false points will be eliminated by using the Kolmogorov-Smirnov statistical test.

- Among the remaining points, only those with the highest curvature are identified as comprising the given fingerprint image's reference point.

The main idea of fingerprint analysis is graphically depicted in Fig. 2. The successive steps of the proposed method are described in detail in the following sections.

\section{Image enhancement and thinning}

In the first stage of our method, the input fingerprint image is enhanced and then thinned. Enhancement leads to an improvement of the image quality. There are many commonly known image preprocessing algorithms for fingerprint enhancement (Jirachaweng et al., 2011; Porwik and Wieclaw, 2008). In our approach, we will employ a method that has previously been well described (Porwik and Wieclaw, 2008). Due to the versatility of this method, it can be applied to any type of fingerprint class. The result of an example input fingerprint image (Fig. 3(a)) enhancement via this method (Porwik and Wieclaw, 2008) is depicted in Fig. 3 (b). After completion of the image quality improvement process, Pavlidis's thinning algorithm was applied (Fig. 3(c)). The image thinning procedure was implemented in Matlab, on the basis of Pavlidis's assumptions.

Hereafter, the pixels of the image being analyzed will be denoted as $I(x, y), x=1, \ldots, W, y=1, \ldots, H$, where $W$ and $H$ represent the dimensions of the image $I$. We assumed that $I(x, y)=1$ for each black pixel, and $I(x, y)=0$ for each white pixel.

\section{Friction ridge extraction strategy}

In the proposed method, each friction ridge of the thinned fingerprint image has to be described by a chain of discrete points $c_{l}=\left(p_{1}, \ldots, p_{n}\right)$, where $n$ is the number of points (pixels) belonging to the $l$-th ridge. For the points $p_{i}$, $i=1, \ldots, n$, their Euclidean coordinates $x$ and $y$ are also recorded. In practice, the chain extracted from a given ridge can be represented as a set of the adjacent pixels on the Euclidean plane. Accordingly, the curvature of any point in the chain can be calculated. From the point of view of the proposed approach, the greatest curvature of every chain is always the most interesting detail.

5.1. Labeling of the pixel-based fingerprint image. In the first step of the friction ridge extraction process, the points of the thinned fingerprint image are labeled according to the following rules:

- label $T$ : points that lie at the beginning or at the end of the ridge.

- label $B$ : points that lie at the bifurcation point of the ridge.

The main idea of the labeling procedure is to count the number $J$ of black pixels placed around the analyzed black pixel $p(x, y)$. This task is accomplished using the following formula:

$$
\begin{aligned}
J(x, y) & =\sum_{a=-1}^{1} \sum_{b=-1}^{1} I(x+a, y+b), \\
x & \in[2, \ldots, W-1], \quad y \in[2, \ldots, H-1] .
\end{aligned}
$$

Depending on the value of $J(x, y)$, the pixel $p(x, y)$ is labeled as a ridge ending point $p^{T}$, or a bifurcation point $p^{B}$ :

$$
p(x, y)= \begin{cases}p^{T}(x, y) & \text { if } J(x, y)=2, \\ p^{B}(x, y) & \text { if } J(x, y)>3 .\end{cases}
$$

It should be noted that, in the image $I$, many $p^{T}$ and $p^{B}$ points can be found. In the proposed approach, each point mentioned will be denoted as $p^{T_{j}}$ or $p^{B_{k}}$, where $j$ and $k$ represent the numbers of a ridge ending or a bifurcation, respectively.

A given entire pixel-based thinned fingerprint image has the form as in Fig. 4(a). In the same figure all possible previously mentioned labels are additionally depicted. In the future, instead of this form, a more compact description will be used in which the pixels that lie between labeled pixels will be approximated by an artificial ridge course. This is shown in Fig. 4(b).

5.2. Friction ridge extraction from a labeled image. Figure 4 shows only one single fingerprint friction ridge. It is obvious that, in practice, a fingerprint image will include many complex friction ridges. The appropriate separation of all these friction ridges is not a trivial task. In our approach we propose two methods: the separation of friction ridges and the determination of the curvature of these ridges. These methods are one of the fundamental components of the proposed approach.

The idea of friction ridge separation will be explained by an example. Let a course of ridges be tagged by labels $T$ or $B$, as shown in Fig. 5(a). The pixel labeling method was previously presented in detail in Section 5.1. 


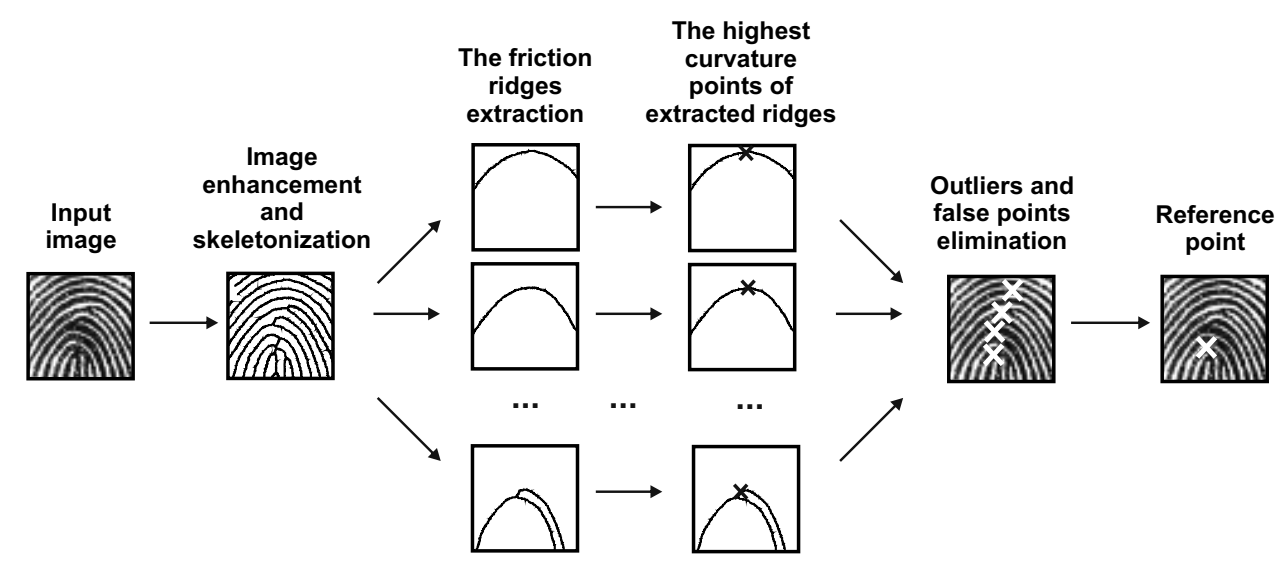

Fig. 2. General idea of the proposed fingerprint reference point detection method.

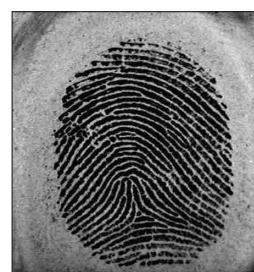

(a)

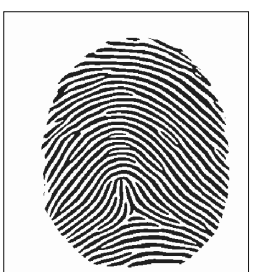

(b)

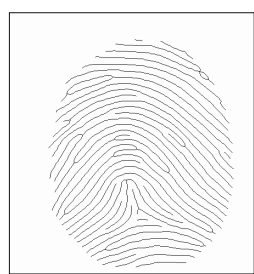

(c)
Fig. 3. Results of preprocessing: an input fingerprint image from an optical sensor (a), the image after quality improvement (b), the image after thinning (c).

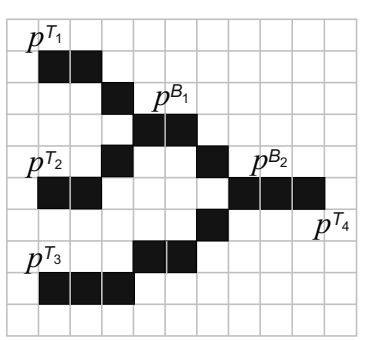

(a)

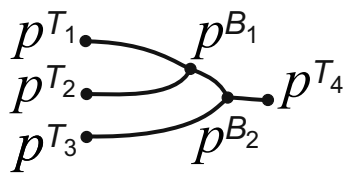

(b)
Fig. 4. Example of a ridge points with different pixel-based labels: $p^{B}$ for bifurcation and $p^{T}$ for terminated pointsthe remainder of the friction ridges pixels remain unlabeled (a), the same data presented in a different form (b).

In the proposed approach, each friction ridge is represented by a chain of points (marked in Fig. 5(b) by gray lines), and each always starts and ends at a $p^{T}$ point. Sometimes a point's chain can consist of sub-chains which pass through labeled points $p^{B}$. For example, the chain $c_{5}$ passes between following points: $p^{T_{1}}, p^{B_{1}}, p^{B_{2}}, p^{T_{4}}$ (Fig.5(b)). In this case, the expanded form of the chain $c_{5}$ can be described by the pixel-based points: $c_{5}=\left(p^{T_{1}}, p_{2}\right.$, $\left.\ldots, p_{i}, p^{B_{1}}, p_{i+2}, \ldots, p_{j}, p^{B_{2}}, p_{j+2}, \ldots, p_{k}, p^{T_{4}}\right)$, where $p_{i}\left(x_{i}, y_{i}\right) \in I$. All sub-chains can be terminated by $T$-type or $B$-type labels. The extraction of all sub-chains from a given fingerprint image is done using our Algorithm 1

Algorithm 1. Collection of the discrete points of the image $I$ that are located on the path between any two labels.

1: input: Thinned fingerprint image $I$ with pixels $p$ labeled by labels $T$ and $B$ type (see Fig. 4). Set of the points of the image with labels $W=$ $\left\{p^{T_{1}}, \ldots, p^{T_{d}}, p^{B_{1}}, \ldots, p^{B_{e}}\right\}$

2: output: Set of sub-chains of the points $U=$ $\left\{u_{1}, \ldots\right\}$, the first and the last point in each sub-chains have label ( $T$ or $B)$;

3: $i=1$;

4: for each unprocessed point $p^{z \in\left\{T_{j}, B_{k}\right\}}, j=$ $\{1, \ldots, d\}, k=\{1, \ldots, e\}$, of the set $W$ do

5: $\quad$ add point $p^{z \in\left\{T_{j}, B_{k}\right\}}$ to the sub-chain $u_{i}$;

6: $\quad p(x, y)=p^{z \in\left\{T_{j}, B_{k}\right\}}$;

7: $\quad$ repeat

8: $\quad$ move the window $S$ from the point $p(x, y)$ to the neighbor black pixel $p^{*}(x, y)$ which does not belong to any sub-chain of the set $U$;

9: $\quad$ add point $p^{*}(x, y)$ to the chain $u_{i}$;

10: $\quad p(x, y)=p^{*}(x, y)$;

11: until the point $p(x, y)$ is not labeled;

12: $\quad i=i+1$;

13: end for

The result of the application of Algorithm 1 to splitting the fingerprint ridge from Fig. 6 a) into a set of sub-chains is shown in Fig. 6(b). At the next stage, the appropriate sub-chains $u_{i} \in U$ are joined together to create a combination of all existing chains belonging to a given friction ridge. The formation of all possible complete chains is achieved by Algorithm A1 (see Appendix). The aim of Algorithm $\mathrm{A} 1$ is to find and then merge together all sub-chains of points, and thus 


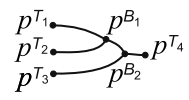

(a)

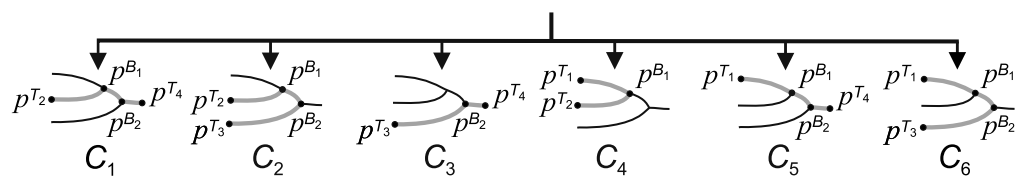

(b)

Fig. 5. Friction ridge separation: the original ridge structure (a), chains $c_{i}$ of discrete points marked by gray lines (b).

create chains of which the first point has the label $T_{i}$ and the last point has the label $T_{j}$.

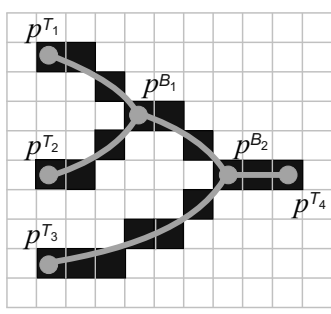

(a)

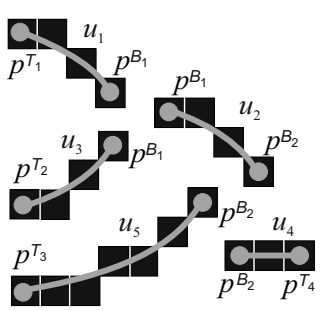

(b)
Fig. 6. Original fingerprint course of ridges (a), ridges after separation by means of Algorithm 1(b).

Finally, all chains found by Algorithm A1 are stored in the set $C$. For example, if we want to find the connections of the sub-chains that lie between points $p^{T_{2}}$ and $p^{T_{3}}$ (see Fig. 6 (a)), then Algorithm A1 generates the result shown in Fig. 77a). It is easy to check that the created chain is the same as the chain $c_{2}$ from Fig. 5 b). Another example of sub-chains connecting $p^{T_{1}}$ and $p^{T_{2}}$ is presented in Fig. 7(b). In practice, Algorithm A1 can be realized by, for example, the Matlab function shortestpathtree (). For the reader's convenience, algorithm pseudo code allows understanding better how the Matlab function works. It also helps in selecting the function parameters.

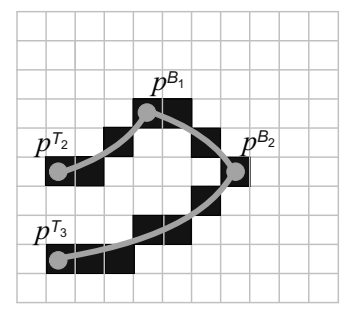

(a)

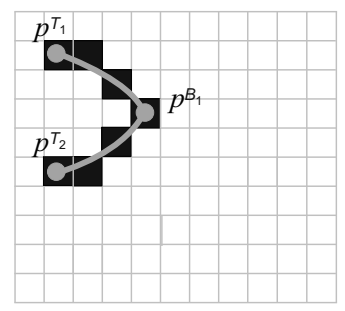

(b)
Fig. 7. Visualization of the chain courses between points: $p^{T_{2}}$, $p^{T_{3}}$ (a), $p^{T_{1}}, p^{T_{2}}$ (b). The chains are determined by Algorithm A1

\section{Determination of the ridge points with the highest curvature}

The next stage of the proposed method is to find the curvatures of all chains $c_{l} \in C, l=1, \ldots, t$ that have previously been determined by Algorithm A1. A single chain $c_{l}=\left(p_{1}, p_{2}, \ldots, p_{n}\right)$ consists of $n$ points (pixels) and is always terminated by $T$-type points. Let $p_{i}$ be an analyzed point of a given chain $c_{l}$. Then, between three selected points, a triangle can be inscribed. The length of the triangle's sides $a$ and $b$ is established on the basis of three conditions, which have to be simultaneously fulfilled:

$$
\begin{gathered}
d_{\min } \leq|a| \leq d_{\max } \\
d_{\min } \leq|b| \leq d_{\max }, \\
1<d_{\min }<d_{\max }<n .
\end{gathered}
$$

The triangles which do meet the conditions (3) are shown in Figs. 8 a) and (b), and the triangles which do not meet the conditions (3) are depicted in Figs. 8(c) and (d).

In practice, the highest ridge curvatures of the previously extracted chains of points are determined by Algorithm A2 (see Appendix).

The optimal values of the parameters $d_{\min }$ and $d_{\max }$ in Algorithm A2 will be calculated in Experiment 2, which will be described in the experimental section.

Finally, we obtain the set $H C$, which comprises $t$ pairs $\left(p_{i}, \alpha_{i}\right)$, where $p_{i}$ denotes the point with the highest ridge curvature $\alpha_{i}$ found on the $i$-th chain of points,

$$
H C=\left\{\left(p_{1}, \alpha_{1}\right),\left(p_{2}, \alpha_{2}\right), \ldots,\left(p_{t}, \alpha_{t}\right)\right\} .
$$

The ridge points with the highest curvature, found by Algorithm A2 on each chain of points, are shown in Fig.9

6.1. Elimination of false points. In most cases, a fingerprint's ridge curvatures increase as they approach the fingerprint's reference points (Kundu and Maiti, 2011; Liu et al., 2005). Unfortunately, in practice, the ridge points with the highest curvature can actually be located far from the reference point, e.g., at the bifurcation points 


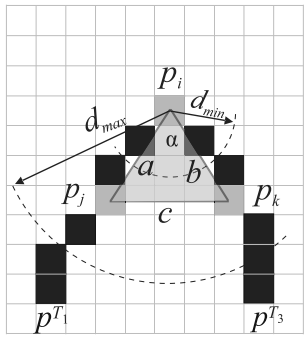

(a)

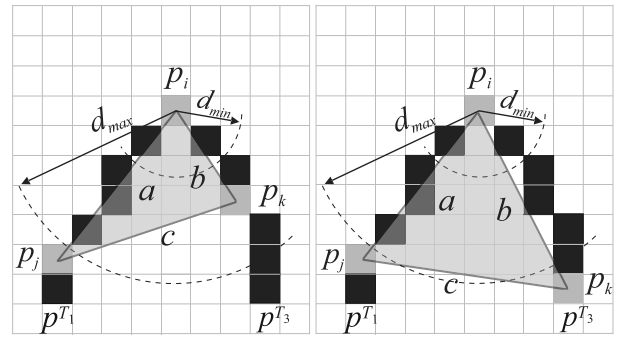

(c)

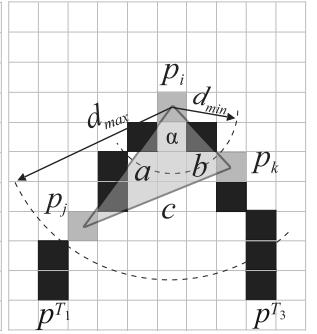

(b)

(d)
Fig. 8. Examples of curvature determination for a given pixeltype fingerprint chain $c_{l}$. Triangles (a) and (b) meet the conditions (3), triangles (c) and (d) do not meet the conditions 3 .

or delta points. Subsequently, in this paper, such points will be called 'false points.'

We have developed a method where false points are efficiently detected and removed from the fingerprint image. Thus, the accuracy of fingerprint reference point detection has been significantly increased. The proposed method consists of two stages:

(a) all ridge points with the highest curvatures but located at the bifurcation points are removed,

(b) the remaining points, for which the curvatures have been determined, are arranged in the ascending order of curvature. Then, for each of the first $N$ points, it is assessed whether or not a given point is located close to, the remaining points. For this purpose, we use the Kolmogorov-Smirnov (K-S) test statistic (Chakravarti et al., 1967). Outliers, identified by the $\mathrm{K}-\mathrm{S}$ test, will not be considered potential reference points. Also, other outlier elimination methods can be applied (Krawczyk, 2016) wherein the outlier datapoints are found by a measure of their local deviations with respect to the outlier's neighbours. Such an approach is algorithmically complex and difficult to interpret. In our case, the proposed Kolmogorov-Smirnov approach is much more beneficial.

6.2. Elimination of the points located at the bifurcation points. All points from the set $H C$ which are located at a distance less than or equal to 5

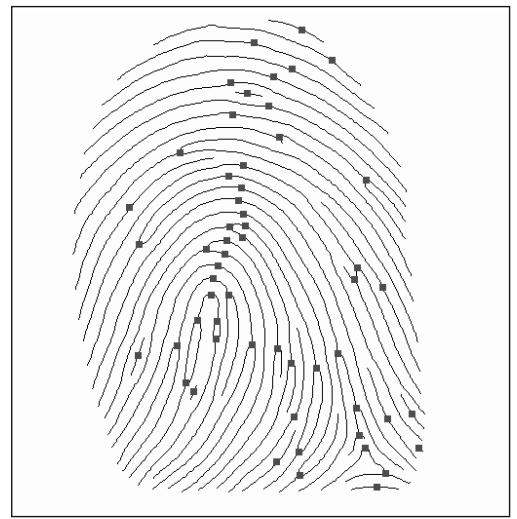

Fig. 9. Fingerprint sample, where points with the highest ridge curvature are indicated by means of Algorithm A2

pixels from any bifurcation $p^{B_{j}}$ will not be taken into account and removed from the image. Information about the bifurcations comes from the set $W=$ $\left\{p^{T_{1}}, \ldots, p^{T_{d}}, p^{B_{1}}, \ldots, p^{B_{e}}\right\}$. Finally, all remaining points $p_{i}$ with the highest curvatures $\alpha_{i}$ are collected into the set $H C^{\prime}$ :

$$
\begin{aligned}
H C^{\prime}= & \left\{\left(p_{i}, \alpha_{i}\right) \in H C: d_{E}\left(p_{i}, p^{B_{j}}\right)>5\right\}, \\
p^{B_{j}} & \in W, \quad i=1, \ldots, t, \quad j=1, \ldots, e,
\end{aligned}
$$

where $d_{E}\left(p_{i}, p^{B_{j}}\right)$ is the Euclidean distance between $p_{i}$ and $p^{B_{j}}$.

The points from the set $H C^{\prime}$ can be directly displayed on a fingerprint image. In Fig. 10, the false points are marked with crosses.

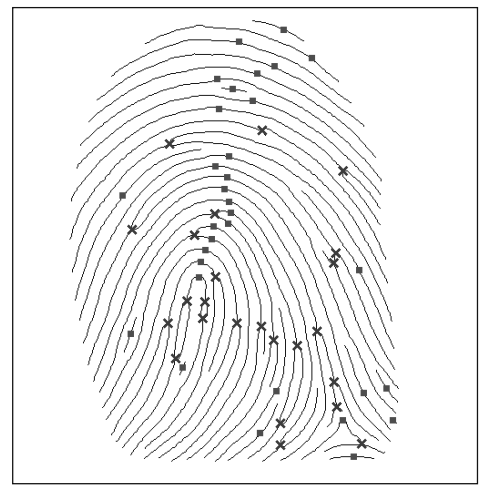

Fig. 10. Processed fingerprint sample with places where false points are marked with crosses. In the future, only potentially correct points (marked with squares) will be taken into consideration.

6.3. Elimination of outliers. This process starts with sorting the points of set $H C^{\prime}$ in ascending order by the curvature magnitude. At the next stage, only $N$ ridges, each including a point with the highest curvature, 
remain in the new set $H C^{\prime \prime}$, so $H C^{\prime \prime} \subset H C^{\prime}$ and $N=\operatorname{card}\left(H C^{\prime \prime}\right)$. Figure 11 shows $N=8$ points with the highest curvature and the values of their curvature. Although the outlier point $p_{1}$ has the highest curvature $\alpha$, that point is not a fingerprint reference point. By means of the K-S test statistic, such outliers will be identified and removed. In the $\mathrm{K}-\mathrm{S}$ test statistic, for every point

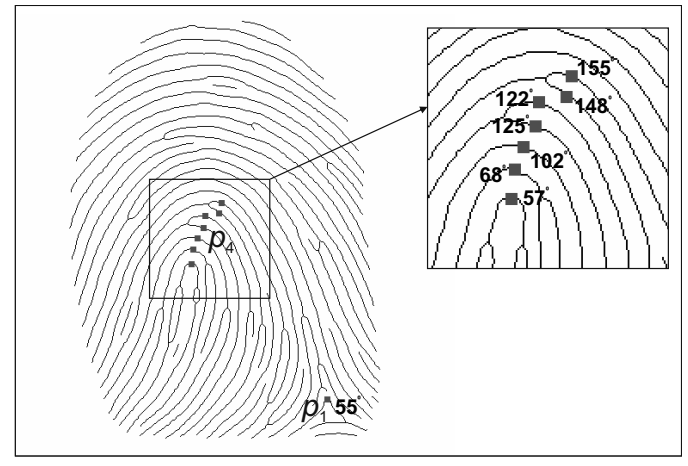

Fig. 11. Processed fingerprint image: its potential reference points and one outlier point.

$p_{i} \in H C^{\prime \prime}$, the cumulative distribution functions (CDFs) $\hat{F}_{1}^{i}(\lambda)$ and $\hat{F}_{2}^{i}(\lambda)$ can be defined as follows:

$$
\begin{gathered}
\hat{F}_{1}^{i}(\lambda)=\frac{1}{N} \sum_{j=1}^{N} \mathbb{1}\left(d_{E}\left(p_{i}, p_{j}\right) \leq \lambda\right), \\
\hat{F}_{2}^{i}(\lambda)=\frac{1}{N^{2}} \sum_{k=1}^{N} \sum_{j=1}^{N} \mathbb{1}\left(d_{E}\left(p_{k}, p_{j}\right) \leq \lambda\right), \\
p_{k}, p_{j} \in H C^{\prime \prime}, \quad \lambda=1,2, \ldots, A, \quad i=1, \ldots, N,
\end{gathered}
$$

where the value $A$ is the length of the diagonal of a given fingerprint image and the $\mathbb{1}(\cdot)$ is the indicator function for the set $H C^{\prime \prime}$ such that

$$
\mathbb{1}\left(d_{E}\left(p_{k}, p_{j}\right) \leq \lambda\right)= \begin{cases}1 & \text { if } d_{E}\left(p_{k}, p_{j}\right) \leq \lambda \\ 0 & \text { otherwise }\end{cases}
$$

Next, for each CDF, we define the value $D_{i} \in[0,1]$ as the maximum vertical distance between $\hat{F}_{1}^{i}(\lambda)$ and $\hat{F}_{2}^{i}(\lambda)$,

$$
D_{i}=\max _{1 \leq \lambda \leq A}\left\|\hat{F}_{1}^{i}(\lambda)-\hat{F}_{2}^{i}(\lambda)\right\|, \quad i=1, \ldots, N .
$$

The CDFs delineated for points $p_{4}$ and $p_{1}$ from Fig. 11 are illustrated Fig. 12

From Fig. 12, we can see that distance $D$ between two CDF is greater for the point $p_{1}$ compared with the point $p_{4}$. For this reason, $p_{1}$ will be treated as an outlier and will not be taken into consideration with regard to reference point detection. Such a conclusion follows directly from the Glivenko-Cantelli theorem (Sharipov, 2011). The practical importance of the theorem comes from the observation that the points located close to each

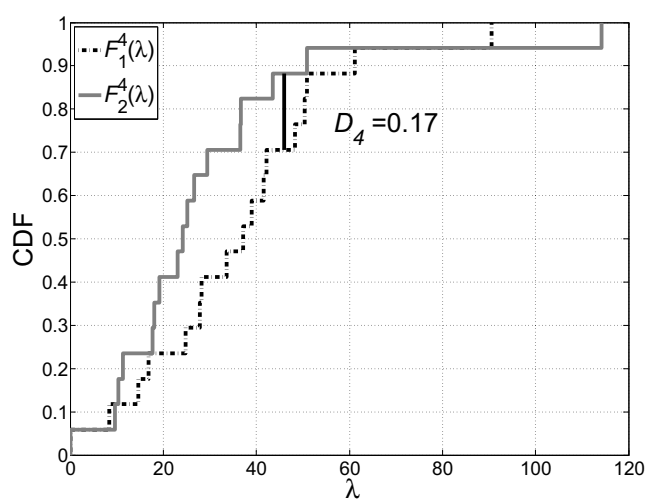

(a)

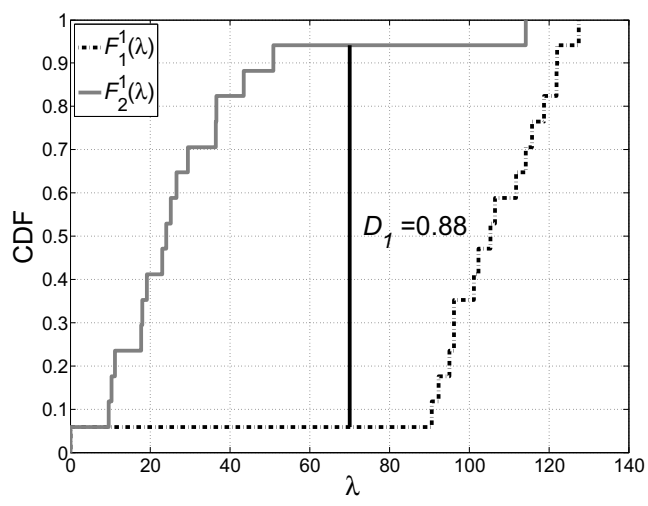

(b)

Fig. 12. CDFs for the points from Fig. 11 for the point $p_{4}$ located close to the remaining points (a), for the outlier point $p_{1}$ (b).

other have a smaller $D$ value compared with those which are further apart. The values of $\left\{D_{1}, . D_{2}, \ldots, D_{N}\right\}$ are calculated separately by means of Algorithm 2 for all $N$ points of the set $H C^{\prime \prime}$.

Based on $\left\{D_{1}, D_{2}, \ldots, D_{N}\right\}$, the set $H C^{\prime \prime \prime}$ is formed as follows:

$$
H C^{\prime \prime \prime}=\left\{\left(p_{i}, \alpha_{i}\right) \in H C^{\prime \prime}: D_{i}<S\right\},
$$

where $S \in[0,1]$ is a threshold value.

The influence of the values of $N$ and $S$ on the removal of false points from the processed fingerprint image will be presented in Experiment 1 in the experimental section.

Finally, the fingerprint reference point $(p, \alpha)_{\text {ref }}$ is selected among the points from the set $H C^{\prime \prime \prime}$ on the basis of the following formula:

$$
\begin{aligned}
(p, \alpha)_{\mathrm{ref}}=\min _{\alpha}\left\{\left(p_{i}, \alpha_{i}\right)\right. & \left.\in H C^{\prime \prime \prime}\right\}, \\
i & =1, \ldots, \operatorname{card}\left(H C^{\prime \prime \prime}\right) .
\end{aligned}
$$




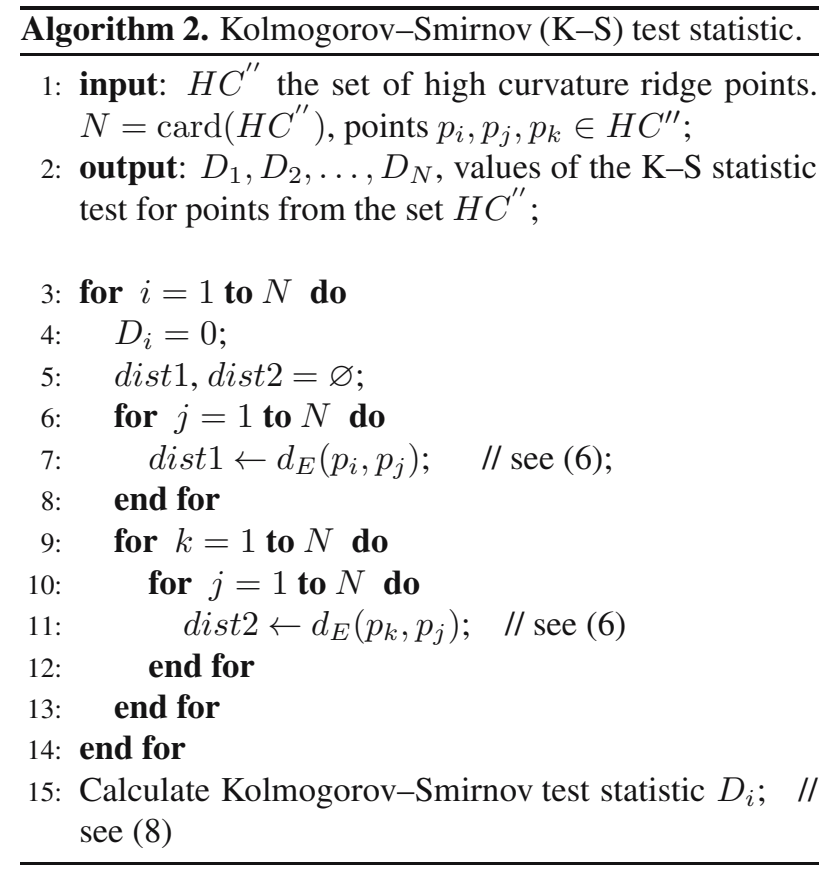

\section{Performed experiments}

The performance of the method for detecting reference points proposed in this paper is evaluated by accuracy coefficients. In the first step, the coordinates of each reference point were manually identified by fingerprint experts. Fingerprint analysis is conducted by experienced fingerprint analysts who follow the accepted description of good practice known as the ACE-V technique (Stevenage and Pitfield, 2016). This acronym describes the four stages of analysis, comparison, evaluation and verification. Thus, expert appraisal is always treated as reference data. The accuracy of an algorithm is determined by comparing it with expert-based reference point estimation. The Euclidean distance between the expert and our algorithm is treated as an error distance of each reference point location.

7.1. Database description. In experiments, we will focus on testing our approach against seven different fingerprint databases. These comprise low-quality as well as high-quality fingerprint images. They allow an analysis of the method and confirm the credibility of the proposed solution. It is worth noting that in some papers experiments were conducted using only a single database (Le and Van, 2012; Liu et al., 2005), so our experiments are a significant extension of those.

Images in the databases have various resolutions and were captured by different fingerprint readers. In our experiments, 4915 images acquired from optical and capacitive scanners were tested. Such a selection is an important factor from a practical point of view because devices equipped with different sensors may have the ability to acquire different types of features. The databases used, the scanners, and image parameters such as image size and resolution are summarized in Table 1

Table 1. Databases employed in the experiments.

\begin{tabular}{|cccc|}
\hline Database & Sensor type & Image size & $\begin{array}{c}\text { Scanner } \\
\text { resolution }\end{array}$ \\
\hline \hline $\begin{array}{c}\text { FVC2000 } \\
\text { DB1 }\end{array}$ & $\begin{array}{c}\text { Low-cost } \\
\text { Optical }\end{array}$ & $300 \times 300$ & $500 \mathrm{dpi}$ \\
\hline $\begin{array}{c}\text { FVC2000 } \\
\text { DB2 }\end{array}$ & $\begin{array}{c}\text { Low-cost } \\
\text { Capacitive }\end{array}$ & $256 \times 364$ & $500 \mathrm{dpi}$ \\
\hline $\begin{array}{c}\text { FVC2002 } \\
\text { DB1 }\end{array}$ & Optical & $388 \times 374$ & $500 \mathrm{dpi}$ \\
\hline $\begin{array}{c}\text { FVC2002 } \\
\text { DB2 }\end{array}$ & Optical & $296 \times 560$ & $569 \mathrm{dpi}$ \\
\hline $\begin{array}{c}\text { FVC2004 } \\
\text { DB1 }\end{array}$ & Optical & $640 \times 480$ & $500 \mathrm{dpi}$ \\
\hline $\begin{array}{c}\text { FVC2004 } \\
\text { DB2 }\end{array}$ & Optical & $328 \times 364$ & $500 \mathrm{dpi}$ \\
\hline NIST & Unknown & $360 \times 364$ & $500 \mathrm{dpi}$ \\
\hline
\end{tabular}

Enlarged samples of fingerprint images and their with reference points are depicted in Fig. 13. The images were captured by different types of fingerprint readers. These images also have hand-applied expert reference points (square) and the points recommended by our procedure (circle) superimposed upon them.
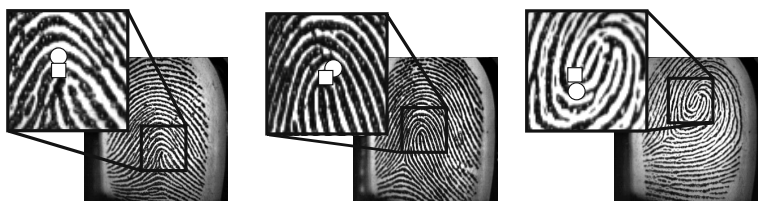

Fig. 13. Reference point localization: fingerprint expert recommendation (square) and the point indicated by our method (circle).

7.2. Precision of fingerprint reference point location. The reference point on a fingerprint image should be located where the fingerprint ridge curvature reaches a local maximum. Reference points are pointed out by means of two methods: (a) manually, by a fingerprint expert, and (b) automatically, by the procedure proposed in this paper. The Euclidean distance between these positions is then computed as the distance error in reference point location. The obtained value is expressed in pixels. The types of distance errors are differently defined across many works (Xie and Zhang, 2016; Weng et al., 2011; Gupta and Gupta, 2016). In out paper, we propose a more restrictive error determination (Le and Van, 2012; Liu et al., 2005), as presented in Table 2. Testing the algorithms on multiple fingerprint databases 
is important in order to ensure the credibility of the tested approach. Thus, we did so in our evaluations.

Table 2. Types of errors of the reference point localization.

\begin{tabular}{|ccc|}
\hline $\begin{array}{c}\text { Type of } \\
\text { error }\end{array}$ & Distance error & Error description \\
\hline Accurate & $\begin{array}{c}\text { Distance error is } \\
\text { not larger than } \\
10 \text { pixels }\end{array}$ & $\begin{array}{c}\text { Error that may be } \\
\text { caused by human } \\
\text { vision. }\end{array}$ \\
\hline Small & $\begin{array}{c}\text { Distance error is } \\
\text { between 10 } \\
\text { pixels and 20 } \\
\text { pixels }\end{array}$ & $\begin{array}{c}\text { Error which may be } \\
\text { caused by both human } \\
\text { vision and algorithm. }\end{array}$ \\
\hline Significant & $\begin{array}{c}\text { Distance error is } \\
\text { between 20 } \\
\text { pixels and } 40 \\
\text { pixels }\end{array}$ & $\begin{array}{c}\text { Error which may have } \\
\text { negative effect on } \\
\text { subsequent processing } \\
\text { steps, but it is still } \\
\text { acceptable. }\end{array}$ \\
\hline $\begin{array}{c}\text { Unaccepted } \\
\text { (critical) }\end{array}$ & $\begin{array}{c}\text { Distance error is } \\
\text { larger than } 40 \\
\text { pixels }\end{array}$ & $\begin{array}{c}\text { Error is not accepted in } \\
\text { fingerprint recognition } \\
\text { systems. }\end{array}$ \\
\hline
\end{tabular}

Benchmark datasets are publicly available. The fingerprints that these contain were collected from realistic environments. These datasets are comprised of fingerprint images captured by various scanners. In our approach, the errors as defined in Table 2 are treated as reference data. The assumptions of Table 2 were adopted with respect to the six available FVC (fingerprint verification competition) and NIST-type (National Institute of Standards and Technology) fingerprint datasets. These were evaluated and compared with other state-of-the-art approaches.

\subsection{Performance evaluations using fingerprint databases.}

Experiment 1. As previously presented, each point from Fig. 11 can be treated as a potential fingerprint reference point. It is obvious that only one of these points should finally be identified out the fingerprint's reference point. Some of these points are outliers and have to be rejected. To accomplish this task, we suggested using the Kolmogorov-Smirnov test statistic. If we studied Eqns. (6) - 9), we would notice that, in the K-S strategy, the values of the two parameters $N$ and $S$ have to be estimated. In our method, the optimal values of these parameters are selected by the particle swarm optimization (PSO) algorithm. PSO is a computational method that iteratively optimizes its solution with regard to a given measure of quality. The use of the PSO method was precisely described in our previous paper (Porwik et al., 2016).

In the proposed experimental scenario, 100 fingerprint images were randomly selected from all the databases listed in Table 1 Each selected image was thinned. Next, the $N$ points were manually marked on every thinned image by a dactyloscopy expert. These $N$ marked points were appropriately divided: $E$ points were treated as outliers if they were located at delta points (if they did occur on the image) or if they were at other, randomly selected, points (but far from the expert's reference point indication). The remainder of $N-E$ points were marked at the fingerprint reference point or close to it. Images with different number of points are depicted Fig. 14. It should be emphasized that in our strategy, deltas are treated as outliers but they do not have to be indicated on the fingerprint image. This means that one advantage of our method is that a broad class of fingerprint images can be processed.

The scenario as mentioned was repeated several times, each time for different values of $N=5,6, \ldots, 15$ and $E=0,1,2$. Finally, 3300 fingerprint images complete with points were prepared.

In the next step, all images were grouped into eleven groups, in such a way that the $N$-th group consists of 300 images with the same value of $N$ and various values of $E$. Afterwards, the optimal values of $S$ in each group were iteratively determined by PSO. The best value of $S$ in each $N$-th group was evaluated by means of the fitness function $f_{N}(S)$, which in our case was as follows:

$$
f_{N}(S)=\frac{\omega}{300}, \quad N=5, \ldots, 15, \quad S \in[0,1],
$$

where $\omega$ denotes the number of images in the $N$-th group from which all $(E)$ outliers were correctly removed by means of Eqn. (9).

The values of the fitness function $f_{N}(S)$ obtained from subsequent PSO iterations are shown in Fig. 15.

From Fig. 15 it follows that the highest $f_{N}(S)$ value was obtained for $N=8$ points. Therefore, the optimal value of the $S$ parameter was checked in successive iterations of the PSO method where $N=8$. The results are shown in Fig. 16

After the 10th PSO iteration, the parameter $S$ becomes stabilized. In subsequent research, only constant values of the parameters $N=8$ and $S=0.39$ will be used in the Kolmogorov-Smirnov test statistic (Eqns. (6)-(9)).

Experiment 2. In Section 6, the highest curvature of the individual fingerprint ridges was evaluated. For this, the values of two parameters $d_{\min }$ and $d_{\max }$ for Algorithm A2 have to be determined. Depending on these parameters, the reference point will be algorithmically determined closer to or further away from the expert's indication. As previously mentioned, the expert's reference point indications are considered to be reference data. Having this reference data, optimal values for the parameters $d_{\text {min }}$ and $d_{\max }$ were determined by means of a grid-search 


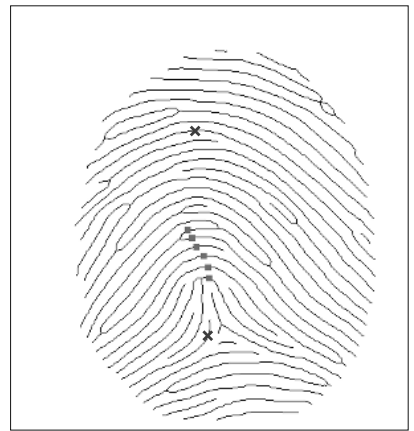

(a)

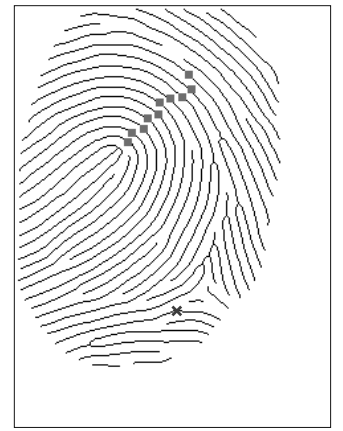

(b)

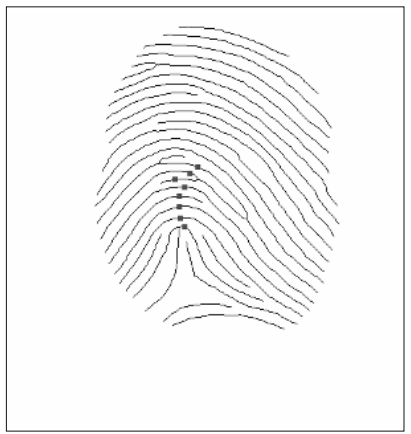

(c)

Fig. 14. Expert's example suggestion of points with the highest ridge curvature for $N=8, E=2$ (a), $N=11, E=1$ (b), $N=8$, $E=0(\mathrm{c})$.

Table 3. Mean distance error and standard deviation of reference point detection relative to the expert.

\begin{tabular}{|lccccc|}
\hline \multicolumn{7}{|c|}{ Mean distance error [px] \pm Std [px] } \\
\hline Database & $\begin{array}{c}\text { Proposed } \\
\text { method }\end{array}$ & $\begin{array}{c}\text { Method 1 } \\
\text { (Porwik and } \\
\text { Wieclaw, 2004) }\end{array}$ & $\begin{array}{c}\text { Method 2 } \\
\text { (Jain } \text { et al., 2000) }\end{array}$ & $\begin{array}{c}\text { Method 3 } \\
\text { (Bo } \text { et al., 2008) }\end{array}$ & $\begin{array}{c}\text { Method 4 } \\
\text { (Bahgat } \\
\text { et al., 2013) }\end{array}$ \\
\hline \hline FVC2000 DB1 & $15.9 \pm 8.39$ & $24.1 \pm 18.53$ & $29.1 \pm 18.24$ & $35.3 \pm 31.03$ & $38.4 \pm 36.62$ \\
FVC2000 DB2 & $15.0 \pm 9.97$ & $35.4 \pm 35.39$ & $39.5 \pm 21.15$ & $39.7 \pm 49.60$ & $37.10 \pm 48.42$ \\
FVC2002 DB1 & $11.9 \pm 8.42$ & $17.7 \pm 11.28$ & $28.9 \pm 21.22$ & $30.6 \pm 44.80$ & $24.4 \pm 20.67$ \\
FVC2002 DB2 & $10.8 \pm 7.85$ & $32.5 \pm 27.25$ & $29.7 \pm 35.7$ & $26.5 \pm 25.46$ & $29.2 \pm 32.13$ \\
FVC2004 DB1 & $10.5 \pm 7.95$ & $16.2 \pm 10.44$ & $24.5 \pm 12.92$ & $27.1 \pm 13.40$ & $21.4 \pm 12.95$ \\
FVC2004 DB2 & $13.0 \pm 8.78$ & $21.2 \pm 17.18$ & $25.4 \pm 12.25$ & $32.3 \pm 46.12$ & $22.7 \pm 29.61$ \\
NIST & $10.2 \pm 9.45$ & $17.8 \pm 19.99$ & $23.2 \pm 13.28$ & $21.7 \pm 41.43$ & $21.4 \pm 27.08$ \\
\hline Mean error & 12.47 & 23.56 & 28.61 & 30.46 & 27.80 \\
Std & 2.26 & 7.61 & 5.42 & 6.00 & 7.31 \\
\hline
\end{tabular}

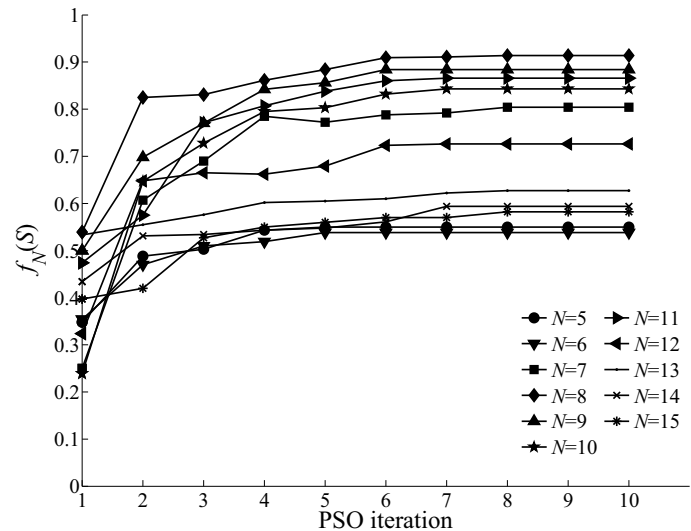

Fig. 15. Fitness function $f$ values for different $N$ and $S$ parameters for various iterations of the PSO algorithm.

procedure. The aim of this procedure was to minimize the distance error between the expert's classification and reference point proposed by our method. Optimal values of both parameters where checked over the range of 2 to 50 points. Figure 17 shows how the distance errors depend on changes in the $d_{\min }$ and $d_{\max }$ values for various fingerprint databases.
The results obtained over the different databases were averaged. Finally, the following values of $d_{\text {min }}=17$ and $d_{\max }=19$ will be used in further experiments.

Experiment 3. In this experiment, we present comparative studies in which we compare various

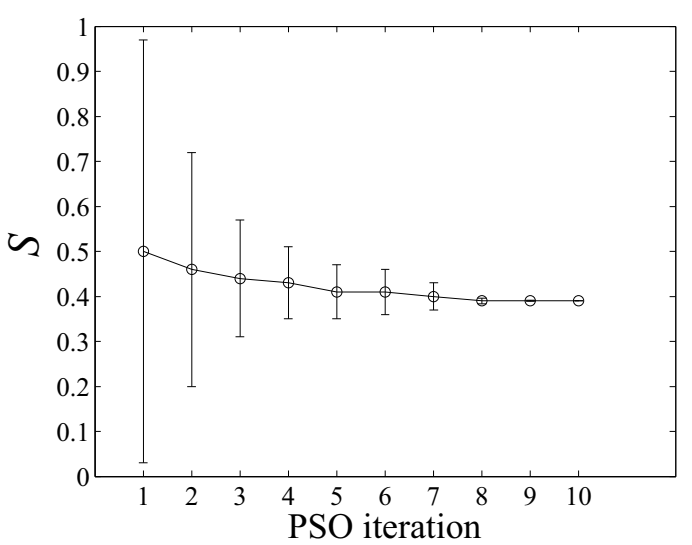

Fig. 16. Changes of the value of the parameter $S$ for subsequent iterations of the PSO strategy carried out for $N=8$ points. 


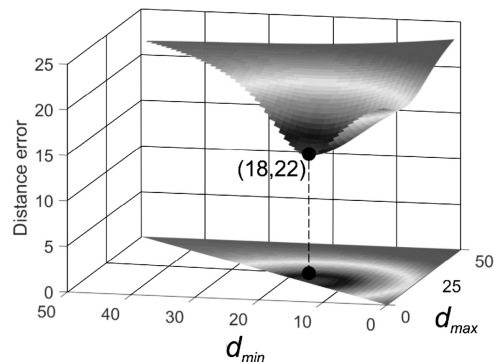

(a)

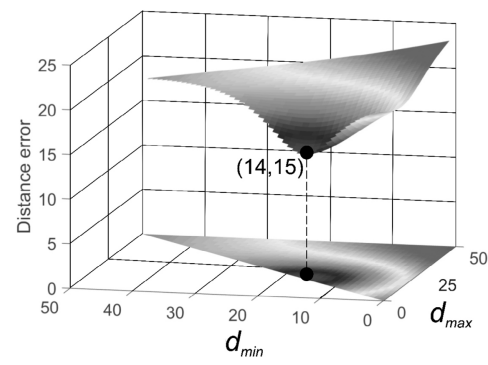

(c)

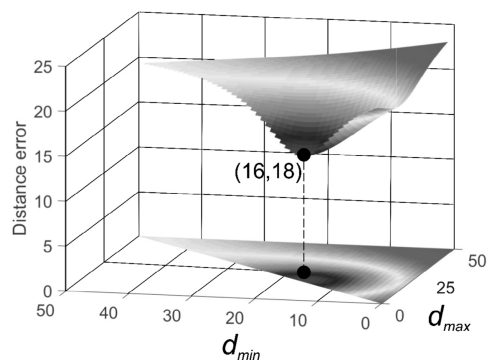

(b)

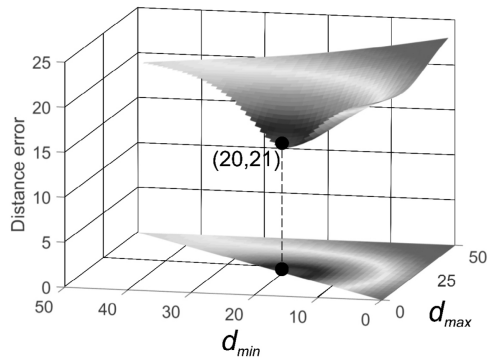

(d)

Fig. 17. Best $d_{\min }\left(d_{\max }\right)$ parameters calculated by a grid-search procedure for various databases: FVC2000 (DB1, DB2) (a), FVC2002 (DB1, DB2) (b), FVC2004 (DB1, DB2) (c), NIST (d).

Table 4. Wilcoxon test to compare our fingerprint reference point detection method with others. The $p$-value and $\left(R^{+} / R^{-}\right)$ranks are performed.

\begin{tabular}{|cccc|}
\hline \multicolumn{4}{|c|}{ Proposed strategy against } \\
\hline $\begin{array}{c}\text { Method 1 } \\
\text { (Porwik and Wieclaw, 2004) }\end{array}$ & $\begin{array}{c}\text { Method 2 } \\
\text { (Jain } \text { et al., 2000) }\end{array}$ & $\begin{array}{c}\text { Method 3 } \\
\text { (Bo } \text { et al., 2008) }\end{array}$ & $\begin{array}{c}\text { Method 4 } \\
\text { (Bahgat } \text { et al., 2013) }\end{array}$ \\
\hline \hline 0.00333 & 0.00333 & 0.00333 & 0.00333 \\
$(0 / 66)$ & $(0 / 66)$ & $(0 / 66)$ & $(0 / 66)$ \\
\hline
\end{tabular}

techniques of the reference point location, and we then show the performance of our proposed strategy. All tests were performed on the basis of complete datasets (see Table 1). These allow a reliable comparison of algorithms and the various databases.

In the comparative studies, we employed the most representative and popular strategies for reference point indication: the Poincaré-based approach (Bo et al., 2008), taxonomies based on the OM, filter banks (Jain et al., 2000), and filter masks (Porwik and Wieclaw, 2004; Bahgat et al., 2013).

In order to demonstrate the effectiveness of our method, the mean distance error and standard deviation (std) between the detected reference points' positions and the expert's indications were computed for each algorithm. The obtained results are given in Table 3

From the results in Table 3, it is evident that the reference point determination strategy proposed in this paper achieves the best performance over all databases used. All reference points, when detected by competitive methods, were located at greater distances from the expert's reference points than the points detected by our method.

In order to fully assess the effectiveness of our method, the results were also statistically evaluated using the Wilcoxon test which, in contrast to the Student test, allows the computation of statistics for data which do not exhibit a normal distribution. The results obtained by the Wilcoxon test for the level of significance $\alpha=0.05$ are summarized in Table 4 $R^{-}$shows the sum of the ranks in favor of our method, whereas $R^{+}$is the sum of the ranks of for the other methods.

The proposed reference point determination strategy gives better results because, for all rows of Table 4 . $R^{-}>R^{+}$and the obtained $p$-values are lower than the level of significance considered. The obtained results are sufficient to conclude that there are statistically significant differences between the approaches shown in Table 3. For all tests, a nominal $p$-value $<0.05$ was considered statistically significant. All of this confirms the hypothesis that our approach generates smaller errors in reference point determination than any of the other 
strategies when evaluated against the same datasets.

Experiment 4. Based on the recommendations from Table 2] it is possible to construct charts for each method which show the influence of distance errors on accuracy rates. A distance error is defined as the Euclidean distance between the reference point as detected by a fingerprint expert and the point as detected by the given method. If the distance error is not larger than a permissible distance, the localization of the reference point is considered to be correct. The permissible distance is predetermined based on the recommendations from Table 2. Performance comparisons are shown in Table 5 and Fig. 18 In the vast majority of cases, our strategy generated a smaller distance error than the other methods.

Experiment 5. In the next experiment, the time complexity of our method was estimated and compared with the results of other strategies. The average reference point localization time per fingerprint was determined for each of the databases listed in Table 1. The results presented in Fig. 19 show that our method is fast and able to be applied to real-time fingerprint recognition. The fastest time for reference point determination was that of Method 4 but, compared to our strategy, that approach generates a significantly greater matching error. As previously mentioned, in our method only a few procedures were used (see Fig. 2), so for each procedure the calculated time can also be separately stated and presented in Table 6

In this experiment, the measurement time was estimated by a PC equipped with an Intel Core i7-3770 processor, $3.40 \mathrm{GHz}, 16 \mathrm{~GB}$ RAM, and Windows 7 x64 operating system. The time taken for the verification phase could easily be improved by using a parallel processing platform, like a graphical processing unit (GPU). This result from the fact that fingerprint ridges can be separately processed, so that parallel implementation of our method is also possible.

Experiment 6. To confirm the quality of our method, additional comparisons with the newest, state-of-the-art approaches were been conducted. These are gathered in Table 7 for different fingerprint databases. This can be done thanks to the use of a wide range of databases in our paper. It is also seen that our strategy of fingerprint reference point localization, gives the better accuracies compared to other solutions regardless of a database.

\section{Conclusions}

In this paper we described a novel method of reference point detection in fingerprint images. The presented method can be useful in practical applications: our numerical results demonstrate a better accuracy of the proposed strategy than that of any of the existing state-of-the-art methods. The advantages of the method can be summarized as follows:

- Experimental results demonstrate that the proposed strategy yields highly accurate reference point localization in digital fingerprint images from benchmark databases. We report that our results only slightly differ from an expert's indications (Table 3). Figure 18, as well as in Tables 5 and 7, we show that our reference point localization accuracy is high and in the range from $93.67 \%$ to $97.50 \%$. Compared with other approaches, our strategy always has a greater precision of reference point localization. It depends on the dataset and was calculated when the error tolerance was expanded from accurate to small errors. Accuracy errors can be caused by imperfection in human vision, with small errors generating the most authoritative results (Le and Van, 2012).

- The proposed strategy of friction ridge curvature determination is conceptually and computationally simpler than other commonly used methods.

- The proposed method is robust to geometric transformations such as rotation and to translations of input fingerprint images. This comes from the ridge curvatures determination method in which the values of the angles and of the formed triangles remain stable.

- Reference point determination is faster compared with the other approaches tested (Fig. 19). Additionally, the proposed approach can be implemented in a parallel environment, because the fingerprint ridges can be analyzed separately. This would further significantly reduce computational time.

\section{References}

Arjona, R., Gersnoviez, A. and Baturone, I. (2011). Fuzzy models for fingerprint description, in A.M. Fanelli et al. (Eds.), Fuzzy Logic and Applications, WILF 2011, Lecture Notes in Computer Science, Vol. 6857, Springer, Berlin/Heidelberg, pp. 228-235.

Bahgat, G., Khalil, A., Abdel Kader, N. and Mashali, S. (2013). Fast and accurate algorithm for core point detection in fingerprint images, Egyptian Informatics Journal 14(1): 15-25.

Bazen, A.M. and Gerez, S.H. (2002). Systematic methods for the computation of the directional fields and singular points of fingerprints, IEEE Transactions on Pattern Analysis and Machine Intelligence 24(7): 905-919.

Bo, J., Ping, T.H. and Lan, X.M. (2008). Fingerprint singular point detection algorithm by Poincaré Index, WSEAS Transactions on Systems 7(12): 1453-1462. 
R. Doroz et al.
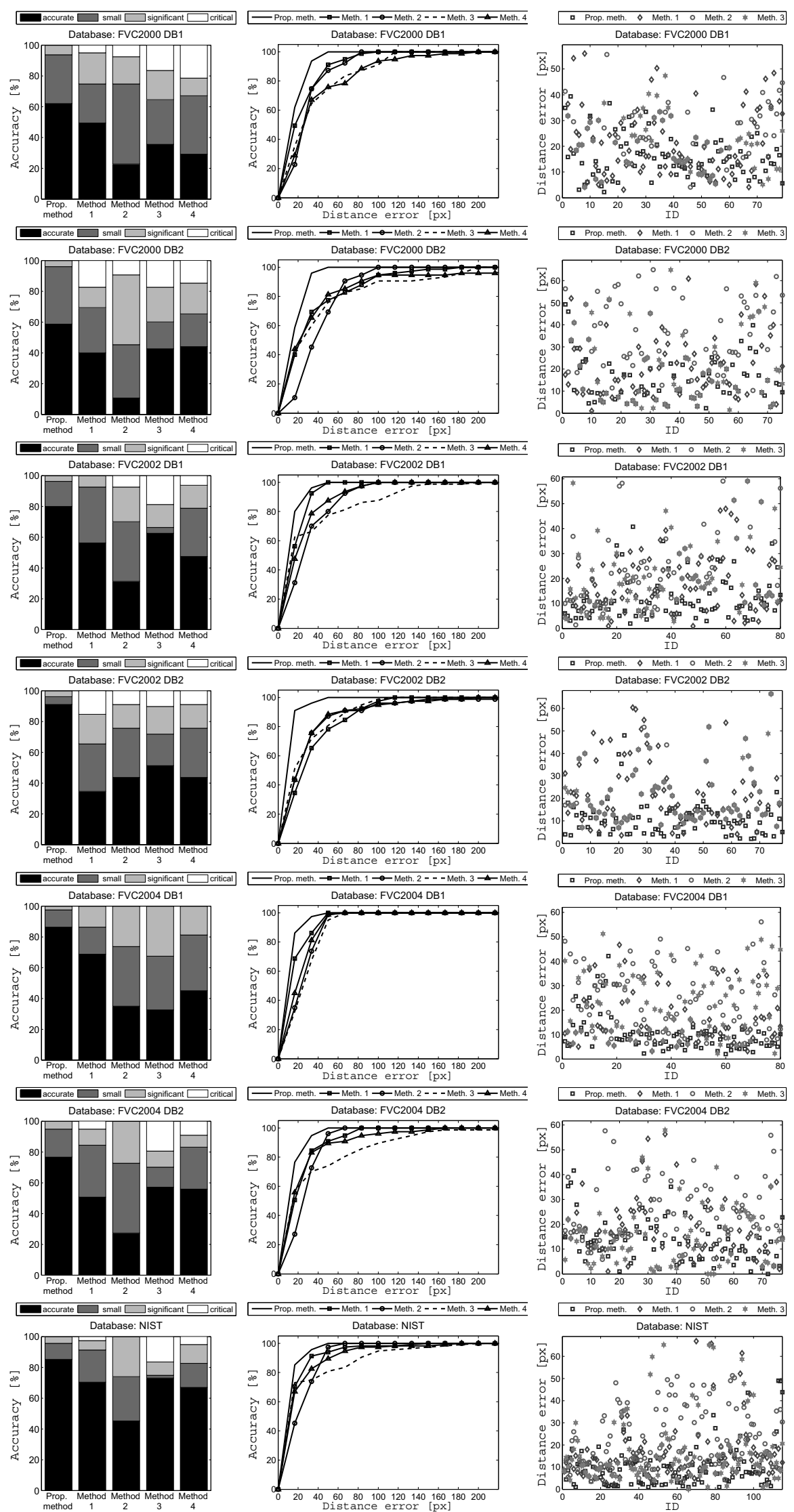

Fig. 18. Performance comparison of different methods of reference point indication, depending on permissible error matching: Method 1 (Porwik and Wieclaw, 2004), Method 2 (Jain et al., 2000), Method 3 (Bo et al., 2008), Method 4 (Bahgat et al., 2013). 
Table 5. Comparison of results for different detection algorithms.

\begin{tabular}{|c|c|c|c|c|c|}
\hline \multicolumn{6}{|c|}{ Accuracy [\%] of fingerprint reference point detection } \\
\hline Database & $\begin{array}{c}\text { Proposed } \\
\text { method }\end{array}$ & $\begin{array}{c}\text { Method 1 } \\
\text { (Porwik and } \\
\text { Wieclaw, 2004) }\end{array}$ & $\begin{array}{c}\text { Method } 2 \\
\text { (Jain } \text { et al., 2000) }\end{array}$ & $\begin{array}{c}\text { Method } 3 \\
\text { (Bo et al., 2008) }\end{array}$ & $\begin{array}{c}\text { Method } 4 \\
\text { (Bahgat } \\
\text { et al., 2013) }\end{array}$ \\
\hline FVC2000 DB1 & 93.67 & 74.68 & 74.68 & $\overline{64.56}$ & 67.09 \\
\hline FVC2000 DB2 & 96.00 & 69.33 & 45.33 & 60.00 & 65.33 \\
\hline FVC2002 DB1 & 96.25 & 92.50 & 70.00 & 66.25 & 78.75 \\
\hline FVC2002 DB2 & 96.15 & 65.38 & 75.64 & 71.79 & 75.64 \\
\hline FVC2004 DB1 & 97.50 & 86.25 & 73.75 & 67.50 & 81.25 \\
\hline FVC2004 DB2 & 94.81 & 84.42 & 72.73 & 70.13 & 83.12 \\
\hline NIST & 95.65 & 91.30 & 73.91 & 74.78 & 82.61 \\
\hline
\end{tabular}

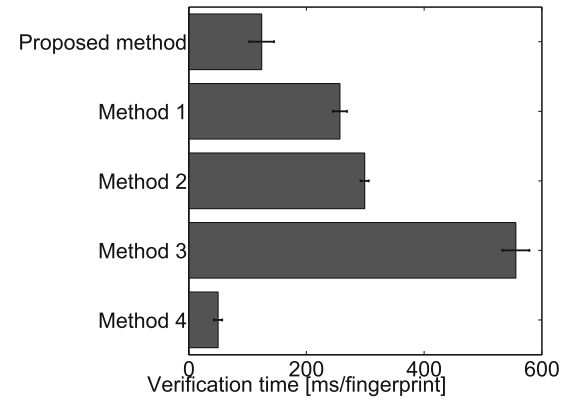

Fig. 19. Average reference point localization time (in milliseconds) per one fingerprint and standard deviation values (visualized by the dash symbol): Method 1 (Porwik and Wieclaw, 2004), Method 2 (Jain et al., 2000), Method 3 (Bo et al., 2008), Method 4 (Bahgat et al., 2013).

Table 6. Execution times of the most important stages in the proposed method.

\begin{tabular}{|l|r|}
\hline Image enhancement & $42 \mathrm{~ms}$ \\
Thinning procedure & $23 \mathrm{~ms}$ \\
Determination of the one chain of points & $1 \mathrm{~ms}$ \\
Determination of the all chains of points & $55 \mathrm{~ms}$ \\
Elimination of false points & $18 \mathrm{~ms}$ \\
Reference point determination & $4 \mathrm{~ms}$ \\
\hline Total & $\mathbf{1 4 3} \mathbf{~ m s}$ \\
\hline
\end{tabular}

Chakravarti, I., Laha, R. and Roy, J. (1967). Handbook of Methods of Applied Statistics, Wiley, New York, NY.

Galar, M., Derrac, J., Peralta, D., Triguero, I., Paternain, D., Lopez-Molina, C., García, S., Benítez, J.M., Pagola, M., Barrenechea, E., Bustince, H. and Herrera, F. (2015). A survey of fingerprint classification. Part I: Taxonomies on feature extraction methods and learning models, Knowledge-Based Systems 81: 76-97.

Gavrilova, M.L. and Monwar, M. (2013). Multimodal Biometrics and Intelligent Image Processing for Security Systems, 1st Edn., IGI Global, Hershey, PA.

Gupta, P. and Gupta, P. (2016). An accurate fingerprint orientation modeling algorithm, Applied Mathematical Modelling 40(15): 7182-7194.
Jain, A.K., Chen, Y. and Demirkus, M. (2007). Pores and ridges: High-resolution fingerprint matching using level 3 features, IEEE Transactions on Pattern Analysis and Machine Intelligence 29(1): 15-27.

Jain, A.K., Prabhakar, S., Hong, L. and Pankanti, S. (2000). Filterbank-based fingerprint matching, IEEE Transactions on Image Processing 9(5): 846-859.

Jin, C. and Kim, H. (2010). Pixel-level singular point detection from multi-scale Gaussian filtered orientation field, Pattern Recognition 43(11): 3879-3890.

Jirachaweng, S., Hou, Z., Yau, W.-Y. and Areekul, V. (2011). Residual orientation modeling for fingerprint enhancement and singular point detection, Pattern Recognition 44(2): 431-442.

Khalil, M.S. (2015). Reference point detection for camera-based fingerprint image based on wavelet transformation, BioMedical Engineering Online 14(1).

Koprowski, R. (2016). Some selected quantitative methods of thermal image analysis in Matlab, Journal of Biophotonics 9(5): 510-520.

Kowal, M. and Filipczuk, P. (2014). Nuclei segmentation for computer-aided diagnosis of breast cancer, International Journal of Applied Mathematics and Computer Science 24(1): 19-31, DOI: 10.2478/amcs-2014-0002.

Krawczyk, B. (2016). Learning from imbalanced data: Open challenges and future directions, Progress in Artificial Intelligence 5(4): 221-232.

Krawczyk, B. and Woźniak, M. (2016). Dynamic classifier selection for one-class classification, Knowledge-Based Systems 107(81): 43-53.

Kundu, M.K. and Maiti, A.K. (2011). Accurate localizations of reference points in a fingerprint image, in S.O. Kuznetsov et al. (Eds.), Pattern Recognition and Machine Intelligence, PReMI 2011, Lecture Notes in Computer Science, Vol. 6744, Springer, Berlin/Heidelberg, pp. 293-298.

Le, T.H. and Van, H.T. (2012). Fingerprint reference point detection for image retrieval based on symmetry and variation, Pattern Recognition 45(9): 3360-3372.

Liu, M., Jiang, X. and Kot, A.C. (2005). Fingerprint reference-point detection, EURASIP Journal on Applied Signal Processing 2005(4): 498-509. 
Table 7. Comparison of different state of the art methods with respect to the fingerprint reference point accuracy factor.

\begin{tabular}{|cccccc|}
\hline Database & $\begin{array}{c}\text { Proposed } \\
\text { method }\end{array}$ & $\begin{array}{c}\text { Nilsson and } \\
\text { Bigun, 2003 }\end{array}$ & $\begin{array}{c}\text { Liu } \text { et al., } \\
2005\end{array}$ & $\begin{array}{c}\text { Le and Van, } \\
2012\end{array}$ & $\begin{array}{c}\text { Zacharias } \\
\text { et al., } 2017\end{array}$ \\
\hline \hline FVC2000 DB1 & $93.67 \%$ & - & - & - & - \\
FVC2000 DB2 & $96.00 \%$ & - & $95.18 \%$ & - & - \\
FVC2002 DB1 & $96.25 \%$ & - & - & - & - \\
FVC2002 DB2 & $96.15 \%$ & - & - & - & $95.81 \%$ \\
FVC2004 DB1 & $97.50 \%$ & $89.87 \%$ & $71.50 \%$ & $96.63 \%$ & $95.19 \%$ \\
FVC2004 DB2 & $94.80 \%$ & - & - & - & - \\
NIST & $95.65 \%$ & - & - & - & - \\
\hline
\end{tabular}

'-' stands for unavailable values

Maltoni, D. (2009). Handbook of Fingerprint Recognition, 2nd. Edn., Springer, London.

Mazurek, P. and Oszutowska-Mazurek, D. (2014). From the slit-island method to the ising model: Analysis of irregular grayscale objects, International Journal Applied Mathematics and Computer Science 24(1): 49-63, DOI: 10.2478/amcs-2014-0004.

Nilsson, K. and Bigun, J. (2003). Localization of corresponding points in fingerprints by complex filtering, Pattern Recognition Letters 24(13): 2135-2144.

Pavlidis, T. (1982). Algorithms for Graphics and Image Processing, Computer Science Press, Rockville, MD.

Porwik, P. and Doroz, R. (2014). Self-adaptive biometric classifier working on the reduced dataset, in M. Polycarpou et al. (Eds.), Hybrid Artificial Intelligence Systems, HAIS 2014, Lecture Notes in Computer Science, Vol. 8480, Springer, Cham, pp. 377-388.

Porwik, P., Doroz, R. and Orczyk, T. (2016). Signatures verification based on PNN classifier optimised by PSO algorithm, Pattern Recognition 60: 998-1014.

Porwik, P., Doroz, R. and Wrobel, K. (2009). A new signature similarity measure, 2009 World Congress on Nature and Biologically Inspired Computing, NABIC 2009, Coimbatore, India, pp. 1022-1027.

Porwik, P. and Wieclaw, L. (2004). A new approach to reference point location in fingerprint recognition, IEICE Electronics Express 1(18): 575-581.

Porwik, P. and Wieclaw, L. (2008). A new efficient method of fingerprint image enhancement, International Journal of Biometrics 1(1): 36-46.

Pujol, F.A., Mora, H. and Girona-Selva, J.A. (2016). A connectionist computational method for face recognition, International Journal of Applied Mathematics and Computer Science 26(2): 451-465, DOI: 10.1515/amcs-2016-0032.

Putz-Leszczyńska, J. (2015). Signature verification: A comprehensive study of the hidden signature method, International Journal of Applied Mathematics and Computer Science 25(3): 659-674, DOI: 10.1515/amcs-2015-0048.

Sharipov, O.S. (2011). Glivenko-Cantelli theorems, in M. Lovric (Ed.), International Encydopedia of Statistical Science, Springer, Berlin/Heidelberg, pp. 612-614.
Srinivasan, V.S. and Murthy, N.N. (1992). Detection of singular points in fingerprint images, Pattern Recognition 25(2): 139-153.

Stevenage, S.V. and Pitfield, C. (2016). Fact or friction: Examination of the transparency, reliability and sufficiency of the ACE-V method of fingerprint analysis, Forensic Science International 267: 145-156.

Tabedzki, M., Saeed, K. and Szczepański, A. (2016). A modified K3M thinning algorithm, International Journal of Applied Mathematics and Computer Science 26(2): 439-450, DOI: 10.1515/amcs-2016-0031.

Wang, Y., Hu, J. and Phillips, D. (2007). A fingerprint orientation model based on 2D Fourier expansion (FOMFE) and its application to singular-point detection and fingerprint indexing, IEEE Transactions on Pattern Analysis and Machine Intelligence 29(4): 573-585.

Weng, D., Yin, Y. and Yang, D. (2011). Singular points detection based on multi-resolution in fingerprint images, Neurocomputing 74(17): 3376-3388.

Xie, S.J. and Zhang, Y. (2016). Beam search algorithm for fingerprint reference point determination based on joint orientation features, International Journal of Science and Research 5(5): 2493-2500.

Zacharias, G.C., Nair, M.S. and Lal, P.S. (2017). Fingerprint reference point identification based on chain encoded discrete curvature and bending energy, Pattern Analysis and Applications 20(1): 253-267.

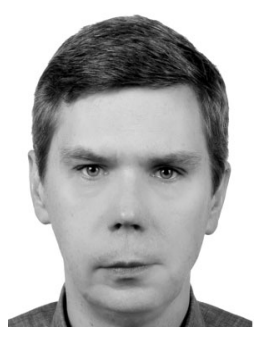

Rafal Doroz received his $\mathrm{PhD}$ degree in computer science from the University of Silesia, Poland, in 2011. Currently, he is an assistant professor in the Computer Systems Department, University of Silesia. He has also been a visiting professor at the University of Calgary, Canada. He main research interests are biometrics, image processing, classifiers, and machine learning. $\mathrm{He}$ has published more than 50 papers in internationals journals and conference materials. 


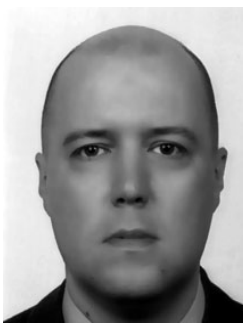

Krzysztof Wrobel received his $\mathrm{MSc}$ and $\mathrm{PhD}$ degrees in computer science from the University of Silesia in 1999 and 2006, respectively. Currently, he works in the Computer Systems Department, Institute of Computer Science, University of Silesia. His research interest areas are biometrics, digital image processing and computer graphics. Dr Wrobel is an author more than 50 scientific papers, published in international journals and conference materials. He also been a visiting professor at the University of Calgary in Canada.

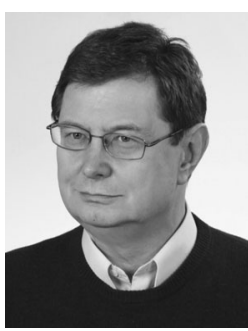

Piotr Porwik is a professor of computer science in the Computer System Department, Computer Science and Materials Science Faculty, University of Silesia, Poland. At present he is the head of the Computer System Department. Currently his scientific researches focus on biometrics, machine learning, classifier systems, image processing and medical imaging. He has published over 100 scientific papers, in Polish and foreign journals as well as numerous national and international conference presentations and talks. He has also published 3 books and edited 5 books. Since 2007 he has been the editor-in-chief of the Journal of Medical Informatics \& Technologies and an associate editor for the International Journal of Biometrics.

\section{Appendix}

Algorithm A1. Formation of the chain $c_{l}$ of points which are terminated by the $T$-type labels.

1: input: Set of the points of the image with the labels $W=\left\{p^{T_{1}}, \ldots, p^{T_{d}}, p^{B_{1}}, \ldots, p^{B_{e}}\right\}$, set of sub-chains of the points $U=\left\{u_{1}, \ldots\right\}$;

2: output: Set $C=\left\{c_{l}\right\}, l=1, \ldots, t$, where $t$ denotes the number of all fingerprint ridges in a given fingerprint image;

3: $l=1$;

4: for each unprocessed pairs $p^{T_{i}}$ and $p^{T_{j}}$ from the set $W$ do

5: $\quad$ if (exist connection between $p^{T_{i}}$ and $\left.p^{T_{j}}\right)$ and $(i<$ j) then

6: $\quad$ create concatenation of sub-chains $u_{i} \in U$, which form continuous chain sequence $c_{l}$ between points $p^{T_{i}}$ and $p^{T_{j}}$;

7: $\quad l=l+1$;

add $c_{l}$ to set $C$;

end if

end for $\overline{\text { Algorithm A2. Highest chain (friction ridge) curvature }}$ determination.

1: input: $C=\left\{c_{1}, . c_{2}, \ldots, c_{t}\right\}$-set of separated chains, $d_{\min }, d_{\max }$ - parameters with arbitrary selected values;

2: output: $H C=\left\{\left(p_{1}, \alpha_{1}\right), \ldots,\left(p_{t}, \alpha_{t}\right)\right\}$ - set of ridge points $p_{i}$ with the highest curvature $\alpha_{i}$;

3: $H C=\varnothing$;

4: for each chain $c_{l}=\left(p_{1}, p_{2}, \ldots, p_{n}\right)$ from the set $C$ do

5: $\quad$ AnglePoint $=\varnothing$;

6: $\quad$ for $i=2$ to $n-1$ do

7: $\quad \alpha_{i}=360^{0}$;

8: $\quad$ for $j=1$ to $i-1$ do

for $k=i+1$ to $n-1$ do

compute the length of the triangle's side $|a|$ between points $p_{i}$ and $p_{j}$; compute the length of the triangle's side $|b|$ between points $p_{i}$ and $p_{k}$; compute the length of the triangle's side $|c|$ between points $p_{j}$ and $p_{k}$; if $|a|,|b| \in\left[d_{\min }, \ldots, d_{\max }\right]$ then

$$
\begin{aligned}
& \alpha=\arccos \left(\frac{|a|^{2}+|b|^{2}-|c|^{2}}{2|a||b|}\right) \cdot \frac{180}{\pi} ; \\
& \text { if }\left(\alpha<\alpha_{i}\right) \text { then } \\
& \quad \alpha_{i}=\alpha ; \\
& \text { end if }
\end{aligned}
$$

end if

\section{end for}

Add the pair $\left(p_{i}, \alpha_{i}\right)$ to the set AnglePoint; end for

\section{end for}

Add to the set $H C$ such the pair $\left(p_{i}, \alpha_{i}\right) \in$ AnglePoint, for which the $\alpha_{i}$ has the smallest value;

\section{4: end for}

Received: 30 March 2017

Revised: 5 July 2017

Accepted: 6 September 2017 